\title{
Anisotropic Dispersion and Partial Localization of Acoustic Surface Plasmons on an Atomically Stepped Surface: Au(788)
}

\author{
M. Smerieri, ${ }^{1}$ L. Vattuone, ${ }^{1,2,{ }^{*}}$ L. Savio, ${ }^{1}$ T. Langer, ${ }^{3}$ C. Tegenkamp, ${ }^{3}$ H. Pfnür, ${ }^{3}$ V. M. Silkin, ${ }^{4,5,6}$ and M. Rocca ${ }^{1,2}$ \\ ${ }^{1}$ IMEM-CNR Unitá Operativa di Genova, Via Dodecaneso 33, 16146 Genova, Italy \\ ${ }^{2}$ Dipartimento di Fisica dell'Universitá di Genova, Via Dodecaneso 33, 16146 Genova, Italy \\ ${ }^{3}$ Institut für Festkörperphysik, Abteilung Atomare und Molekulare Strukturen, Leibniz Universität Hannover, \\ Appelstrasse 2, D-30167 Hannover, Germany \\ ${ }^{4}$ Departamento de Física de Materiales, Facultad de Ciencias Químicas, Universidad del País Vasco, \\ Apartado 1072, 20080 San Sebastián/Donostia, Spain \\ ${ }^{5}$ Donostia International Physics Center (DIPC), Paseo de Manuel Lardizabal 4, 20018 San Sebastián/Donostia, Spain \\ ${ }^{6}$ IKERBASQUE, Basque Foundation for Science, 48011 Bilbao, Spain
}

(Received 21 May 2014; published 29 October 2014)

\begin{abstract}
Understanding acoustic surface plasmons (ASPs) in the presence of nanosized gratings is necessary for the development of future devices that couple light with ASPs. We show here by experiment and theory that two ASPs exist on $\mathrm{Au}(788)$, a vicinal surface with an ordered array of monoatomic steps. The ASPs propagate across the steps as long as their wavelength exceeds the terrace width, thereafter becoming localized. Our investigation identifies, for the first time, ASPs coupled with intersubband transitions involving multiple surface-state subbands.
\end{abstract}

DOI: 10.1103/PhysRevLett.113.186804

Acoustic surface plasmons (ASPs) originate from the excitation of a 2D electron gas whenever it is effectively screened by an underlying 3D electron gas [1]. Contrary to sheet plasmons, characterized by a square-root-like dependence on the wave vector [2], ASPs have a linear soundlike dispersion and hence frequency-independent group and phase velocities. These excitations are very promising for future applications in plasmonics because the speed, and thus the wavelength, of ASPs is 3 orders of magnitude lower than that of light, permitting us in principle to locate plasmonic excitations on the scale of a few nanometers. A hypothetical polychromatic ASP signal would indeed propagate without distortion, allowing for accurate signal processing.

Suitable 2D electron gases are associated with the Shockley surface state (SSS) or any other 2D free electron gas. Linearly or quasilinearly dispersing ASPs have indeed been reported for bare and O-covered $\mathrm{Be}(0001)$ [3,4], $\mathrm{Cu}(111)$ [5,6], and $\mathrm{Au}(111)[7,8]$, as well as for graphene deposited on metal substrates. Generally speaking, they are expected to exist whenever two slightly spatially separated electron gases interact sufficiently strongly $[9,10]$. Ab initio density-response calculations $[3,7,11]$ confirmed their existence on $\mathrm{Be}, \mathrm{Cu}, \mathrm{Ag}$, and $\mathrm{Au}$ surfaces.

The dispersion curves of ASP and light do not, however, per se cross at clean surfaces, so a momentum source is needed to realize coupling of the ASP with photons; this can be realized by, e.g., a grating. However, the short wavelength of the ASP requires realization of this grating on the atomic scale. For example, it can be generated by a regular array of atomic steps, as provided by vicinal surfaces of single crystals. Nanostructuring, however, implies concomitant generation of atomic defects, so it
PACS numbers: 78.68.+m, 73.20.At, 73.20.Mf, 79.20.Uv

is necessary to understand how defects and confinement in nanosized regions modify the ASP dispersion. As a first step, we recently demonstrated that the ASP survives damage induced by heavy ion bombardment on $\mathrm{Cu}(111)$ [12], at least as long as the SSS itself survives the treatment. The question we address here is how the plasmonic structure of a bare $\mathrm{Au}(111)$ surface is transformed by a regular array of atomic steps. Combining experiment and density-response calculations, we demonstrate the existence of two plasmonic modes at the $\mathrm{Au}(788)$ surface both parallel and perpendicular to the steps, due to the splitting of the SSS band into separate subbands caused by the step potential.

On vicinal surfaces the wave functions of the electronic states are scattered by regularly spaced atomic arrays that may confine them in well-defined quantum wells $(\mathrm{QW})$. This is also expected for a SSS [13]. Because the slope of the ASP dispersion is, as a first approximation, proportional to the Fermi velocity, the ASP is directly affected. Indeed, quantum confinement and energy gap opening become visible on stepped $\mathrm{Au}$ and $\mathrm{Cu}$ surfaces once step-step interactions become sufficiently small [13-15]. For stepped $\mathrm{Au}(111)$ this occurs for terrace widths exceeding $d_{c}=$ $20 \AA$ [16]. From a simple theory one would expect a similar gap opening in the ASP dispersion. However, this model has to be strongly modified for stepped surfaces. As we show, it is only valid for wavelengths much smaller than the step separation, whereas at longer wavelengths the potential modulation by the steps is weak enough for the plasmons to still effectively propagate. We therefore studied the electronic excitations on $\mathrm{Au}(788)$ by energy loss spectroscopy-low energy electron diffraction (ELSLEED) [17-19]. This surface consists of (111)-oriented 

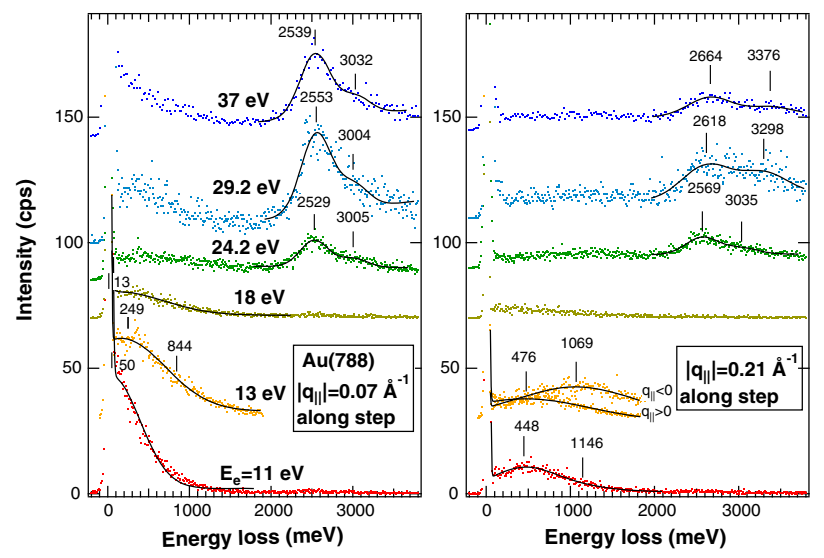

FIG. 1 (color online). ELS-LEED spectra recorded for two fixed momentum exchanges $\left(0.07 \AA^{-1}\right.$ and $\left.0.21 \AA^{-1}\right)$ in the direction along the steps and parametric in $E_{e}$. Three losses are apparent. Inspection of the loss values observed under different scattering conditions (see spectra at $13 \mathrm{eV}$ in the right panel) indicates that at least two different losses contribute to the low energy-loss range.

terraces of width $d$ about $40 \AA$, i.e., larger than $d_{c}$, separated by monatomic close-packed steps. Au was chosen for its chemical inertness and, thus, its importance for future ASP devices.

In Fig. 1 we report ELS-LEED spectra recorded for two different $q_{\|}$in the direction along the steps and for different $E_{e}$. The losses are present in the energy regions around $2.5 \mathrm{eV}$ and below $1 \mathrm{eV}$. The former corresponds to the excitation of the conventional optical surface plasmon [20], which shows the well-known positive dispersion characteristic of noble metals $[8,21,22]$. We will not discuss it further here, but note only that its intensity is largest at the highest $E_{e}$. As expected for a metallic system, the inelastic intensity in the very low electron energy loss (EEL) region is mainly associated with the Drude tail [23]. At low $E_{e}$, on the other hand, this plasmon is barely visible, while a broad maximum is present in the low-frequency region, as found in our previous investigations of the ASP on different metals. The ELS maximum depends nonmonotonically on $E_{e}$ and the scattering geometry, typical for impact scattering [24] and consistent with the multipolar distribution of the excited charges (see Supplemental Material [25]).

Figure 2 reports spectra recorded at $E_{e}=11 \mathrm{eV}$ for various $q_{\|}$both across (red, left panel) and along (blue, right panel) the steps. Figure 3 shows similar data recorded with $E_{e}=13 \mathrm{eV}$. It is apparent that in both cases we excite $q_{\|}$-dependent features. At any given $q_{\|}$the loss is definitively smaller at $E_{e}=11 \mathrm{eV}$ than at $E_{e}=13 \mathrm{eV}$ and, at $E_{e}=11 \mathrm{eV}$, an evident anisotropy is present between the direction across (Fig. 2, left panel) and along (Fig. 2, right panel) the steps. It is therefore again evident that different modes are present whose relative cross section depends on $E_{e}$ and the scattering geometry, confirming their nondipolar origin already highlighted in the discussion of Fig. 1.

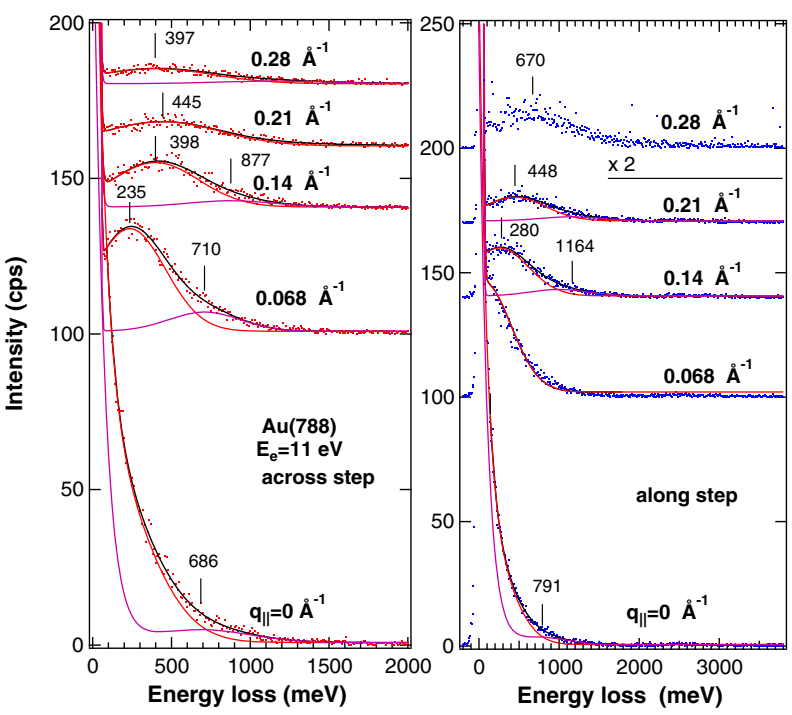

FIG. 2 (color online). ELS-LEED spectra recorded for various $q_{\|}$values across (left) and along (right) the steps. The ELS maxima are anisotropic with respect to the direction of $q_{\|}$.

The position of the energy losses was determined by a multicomponent fit of the EEL spectra, including a negative exponential mimicking the Drude tail and two Gaussians describing the two low-frequency losses. The resulting peak positions are collected vs exchanged momentum in Fig. 4. Minor peaks appearing as shoulders in the spectra (see, e.g., the component at $844 \mathrm{meV}$ in the $E_{e}=13 \mathrm{eV}$ spectrum in Fig. 1, left panel) are omitted, since they are not sufficiently well defined. Our data clearly indicate that two losses with soundlike dispersion are present, denoted by I and II in Fig. 4(a). Across the steps the data points level out at large $q_{\|}$. The branch with the steeper slope originates clearly from the ASP associated to the SSS of the (111) terraces, which has a very similar slope as on flat $\mathrm{Au}(111)$ (solid black line [7]), while the lower branches have no

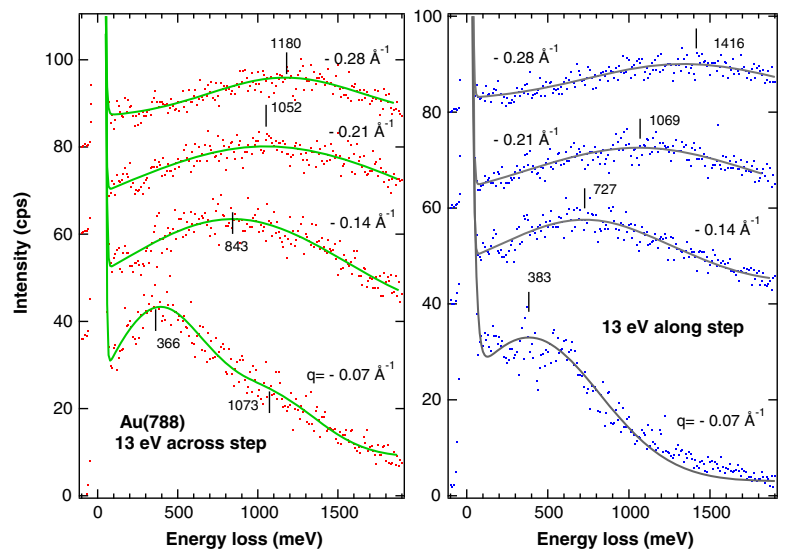

FIG. 3 (color online). Same as Fig. 2 for $13 \mathrm{eV}$. Note the definitively higher energy losses at any given $q_{\|}$compared to Fig. 2. 

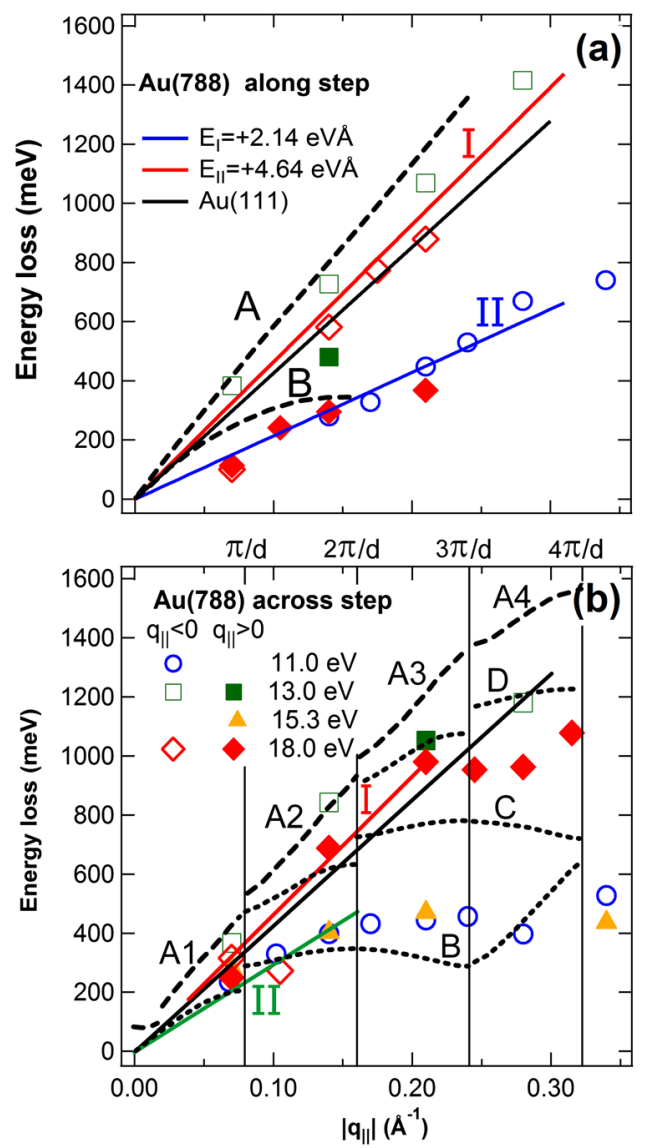

FIG. 4 (color online). EEL peak positions, as measured at room temperature by ELS-LEED on $\operatorname{Au}(788)$ (a) along and (b) across the steps, vs exchanged in-plane momentum $q_{\|}$and comparison with theory. The red lines correspond to the linear best fit to the isotropic higher-energy branch. Blue and green lines are a similar fit for the lower-energy branch along and across the steps, respectively, where across the steps the fit was limited to $q_{\|}<0.16 \AA^{-1}$. Open and filled symbols correspond to experimental data collected, respectively, at positive and negative $q_{\|}$. The solid black line is the dispersion of the Au(111) ASP [7]. Dashed and dotted lines indicate the theoretical plasmon-mode dispersion (see text).

counterpart on pristine $\mathrm{Au}(111)$. Fitting the data with linear functions, we obtain an intercept at the origin compatible with zero within 2 standard deviations. Rerunning the fit with the constraint of zero energy loss at vanishing momentum transfer, we obtain along the steps slopes of 4.64 and $2.14 \mathrm{eV} \AA$ for modes I and II, respectively. Across the steps, we obtain nearly the same slope for the upper branch and the larger value of $2.94 \mathrm{eV} \AA$ for the lower one.

In order to model the physical scenario on the (788) surface we took into account the transformation of the $\mathrm{Au}$ (111) SSS band into the QW subbands observed in photoemission experiments [13]. First, a nearly free electron gas model is employed to accurately reproduce the $\mathrm{Au}$ (111) SSS dispersion (see Fig. S2 in the Supplemental Material [25]), presenting deviations from a perfect parabolic shape above the Fermi level, as inferred from

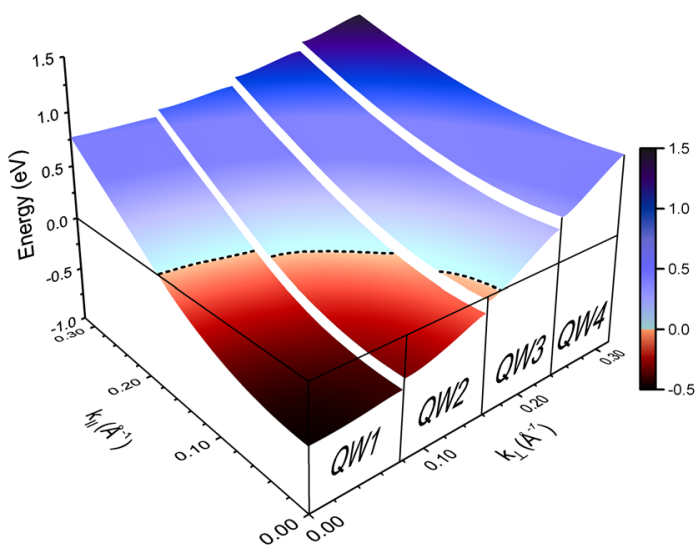

FIG. 5 (color online). Surface-state dispersion in the extended band scheme for $\mathrm{Au}(788)$ calculated for the 1D model and used to fit the photoemission data of Ref. [13]. The dotted lines show the Fermi level in each quantum-well subband.

scanning tunneling spectroscopy (STS) experiments [27] and previous ab initio band-structure calculations $[7,28]$. Second, a simple but realistic step potential (a plateau for terraces and a short-range reflective potential at steps) similar to that proposed in Ref. [13] was used to obtain the SSS subbands. The resulting electronic structure in the extended band scheme is shown in Fig. 5, where one can observe the opening of energy gaps in the direction across the steps. We find that only two QW subbands are significantly occupied and produce relevant effects on the excitation spectrum. Therefore, we shall consider QW3 as totally unoccupied. This electronic structure was then used to perform charge-density response calculations, the details of which are given in the Supplemental Material [25]. These calculations produced the loss function and, from its peaks, information about the collective excitations in the system was retrieved.

The calculated plasmon peak dispersion along the steps is shown in Fig. 4(a) by dashed lines. Single-particle and collective excitations can be distinguished from the form of the dielectric function $\epsilon$, which in the latter case shows a zero crossing in the real part associated with a minimum in the imaginary part allowing us to identify unambiguously the QWs from which they originate. We interpret mode $A$ along the steps, which closely follows the experimental peak I, as an intrasubband plasmon associated with the QW1 subband, characterized by the fastest Fermi velocity in this direction, see Fig. 5. Hence, plasmon mode I results from incomplete screening by carriers within this subband of the slower-moving bulk carriers as it occurs at $\mathrm{Au}(111)$ [7]. Its slope is very close to that of the ASP on $\mathrm{Au}(111)$ because of the relatively small distortion of the SSS dispersion at the wave vectors corresponding to the QW1 subband. Despite rather small energy gaps between the QW subbands, the effect is strong enough to give rise to a second, weaker peak $B$ at lower energies. This additional intrasubband plasmonic peak, correlating with the experimentally observed branch II, is associated with the QW2 
subband, characterized by the slowest Fermi velocity along the steps. Hence, we interpret it as an ASP resulting from the dynamical screening of the slower carriers in QW2 by those moving faster at the Fermi level in QW1. The calculated initial slope of the dispersion of peak $B$ is, however, almost twice as large as in experiment, and theory cannot reproduce its dispersion for $q_{\|}>0.15 \AA^{-1}$. We expect that, in order to obtain reliable information on both the energy dispersion and width of this as well as of other modes, a full inclusion of the $a b$ initio electron band structure is required $[3,7,11]$, which was computationally not feasible for $\mathrm{Au}(788)$.

Across the steps, the situation is even more complex due to the possibility of numerous intersubband transitions involving different QW subbands. As seen in Fig. 4(b), at $q_{\|}<0.16 \AA^{-1}$ the experiment shows two dispersing modes: mode I, traced down to an energy of $\sim 300 \mathrm{meV}$, and mode II, observed down to $\sim 200 \mathrm{meV}$. At large $q_{\|}$'s the dispersion of both modes flattens out. The calculations reveal that at vanishing $q_{\|}$'s the mode $A 1$, corresponding to the experimental mode $\mathrm{I}$, has an energy threshold at about $100 \mathrm{meV}$. It is an intersubband plasmon involving the QW1, QW2, and QW3 subbands. In the larger momentum region such a mode (denoted by the separate $A 2, A 3$, and $A 4$ branches) is an intersubband plasmon associated with the intersubband transitions from the initial states within the occupied part of the QW1 subband into the final states in the QW3, QW4, and QW5 subbands, respectively. Calculations predict that minigaps exist in its dispersion at the Brillouin zone boundaries of the superlattice, thereby opening the possibility of exciting the ASP by light. However, these gaps might be obscured due to lifetime broadening (underestimated by current calculations), which is expected to be similar to that found by $a b$ initio calculations for $\mathrm{Au}(111)$ [7], in agreement with our experiments. As evident from the high resolution EEL spectra, the width of the peaks is comparable with their energy, thus precluding the observation of small gaps in the dispersion. Indeed the experimental FWHM is comparable to that observed for $\mathrm{Au}(111)$ [7], implying that steps do not significantly affect the lifetime of the excitation. Additionally, theory predicts weaker plasmon peaks at $q_{\|}>0.08 \AA^{-1}$, denoted $C$ and $D$, whose dispersion shows breaks at $q_{\|}$values that are multiples of $\pi / d$. The measured peak I results therefore likely from such unresolved contributions. Mode $C$ is associated with the QW2 $\rightarrow$ QW4 intersubband transitions, whereas mode $D$ is determined by the intersubband transitions involving several QW subbands, which makes an assignment of its origin more dubious. The manifold of these plasmonic excitations is reflected in the experimental loss spectra as a broad peak with flat dispersion centered at $1050 \pm 80 \mathrm{meV}$.

At small $q_{\|}$'s the experimental lower-energy mode II corresponds to the calculated acousticlike plasmon mode $B$.
It is associated with the intrasubband transitions within the QW2 subband, which has the fastest carriers at the Fermi level in this direction. As seen in Fig. 4(b), at larger $q_{\|}$'s the experimental dispersion of mode I levels off (at $420 \pm 50 \mathrm{meV}$ ). According to theory, in the $0.16-0.24 \AA^{-1}$ momentum range this mode is an intersubband plasmon involving the QW1 $\rightarrow$ QW2 transitions, whereas at larger $q_{\|}$the relevant intersubband transitions are QW2 $\rightarrow$ QW3. The calculations show that the collective response of the QW1 subband in this direction is too weak to be experimentally detected.

In conclusion, these findings lead to the following remarkable physical scenario: (a) Along the steps we observe two ASP modes with different group velocities determined by the maximal Fermi velocities of two partly occupied SSS subbands. (b) The anisotropy introduced by the steps on $\mathrm{Au}(788)$ does not automatically lead to plasmon localization normal to the terraces, e.g., as found for the Ag monolayer on $\mathrm{Si}(557)$ [29], but the SSS subband formation is reflected by a splitting of the ASP also normal to the steps. (c) The QW1 and QW2 subbands are still able to generate propagating plasmonic modes across the steps at wavelengths longer than the terrace width. The slightly different slopes of the modes parallel and normal to the steps reflect the anisotropy of the system. (d) Across the steps, signs of plasmon localization become visible when $q_{\|}$exceeds $G_{0}=0.078 \AA^{-1}$, the reciprocal lattice vector of the periodic step array, and become increasingly dominant when multiples of $G_{0}$ can be transferred. Together with an increasing efficiency of momentum transfer at steps with decreasing plasmon wavelength [19], this leads to nearly complete localization at the highest $q_{\|}$of our measurements.

We acknowledge J. E. Ortega for discussions and for the loan of the $\mathrm{Au}(788)$ sample used in the present experiment. We thank Compagnia di San Paolo and the Deutsche Forschungsgemeinschaft for funding.

* Corresponding author. vattuone@fisica.unige.it

[1] V. M. Silkin, A. García-Lekue, J. M. Pitarke, E. V. Chulkov, E. Zaremba, and P. M. Echenique, Europhys. Lett. 66, 260 (2004).

[2] F. Stern, Phys. Rev. Lett. 18, 546 (1967).

[3] B. Diaconescu et al., Nature (London) 448, 57 (2007).

[4] M. Jahn, M. Müller, M. Endlich, N. Néel, J. Kröger, V. Chis, and B. Hellsing, Phys. Rev. B 86, 085453 (2012).

[5] K. Pohl, B. Diaconescu, G. Vercelli, L. Vattuone, V. M. Silkin, E. V. Chulkov, P. M. Echenique, and M. Rocca, Europhys. Lett. 90, 57006 (2010).

[6] J. Pischel, E. Welsch, O. Skibbe, and A. Pucci, J. Phys. Chem. C 117, 26964 (2013).

[7] L. Vattuone, M. Smerieri, T. Langer, C. Tegenkamp, H. Pfnür, V. M. Silkin, E. V. Chulkov, P. M. Echenique, and M. Rocca, Phys. Rev. Lett. 110, 127405 (2013). 
[8] S. J. Park and R. E. Palmer, Phys. Rev. Lett. 105, 016801 (2010).

[9] A. Politano, A. R. Marino, V. Formoso, D. Farías, R. Miranda, and G. Chiarello, Phys. Rev. B 84, 033401 (2011).

[10] H. Pfnür, T. Langer, J. Baringhaus, and C. Tegenkamp, J. Phys. Condens. Matter 23, 112204 (2011).

[11] J. Yan, K. W. Jacobsen, and K. S. Thygesen, Phys. Rev. B 86, 241404(R) (2012).

[12] L. Vattuone, G. Vercelli, M. Smerieri, L. Savio, and M. Rocca, Plasmonics 7, 323 (2012).

[13] J. E. Ortega, M. Ruiz-Osés, J. Cordón, A. Mugarza, J. Kuntze, and F. Schiller, New J. Phys. 7, 101 (2005).

[14] F. Schiller, M. Corso, J. Cordón, F. J. Garcia de Abajo, and J. E. Ortega, New J. Phys. 10, 113017 (2008).

[15] M. Corso, F. Schiller, L. Fernandez, J. Cordón, and J. E. Ortega, J. Phys. Condens. Matter 21, 353001 (2009).

[16] J. E. Ortega, A. Mugarza, V. Pérez-Dieste, V. Repain, S. Rousset, and A. Mascaraque, Mater. Sci. Eng. B 96, 154 (2002).

[17] H. Claus, A. Büssenschütt, and M. Henzler, Rev. Sci. Instrum. 63, 2195 (1992).

[18] V. Zielasek, N. Rönitz, M. Henzler, and H. Pfnür, Phys. Rev. Lett. 96, 196801 (2006).
[19] T. Langer, J. Baringhaus, H. Pfnür, H. W. Schumacher, and C. Tegenkamp, New J. Phys. 12, 033017 (2010).

[20] R. H. Ritchie, Phys. Rev. 106, 874 (1957).

[21] M. Rocca, Surf. Sci. Rep. 22, 1 (1995).

[22] S. J. Park and R. E. Palmer, Phys. Rev. Lett. 102, 216805 (2009).

[23] B. N. J. Persson and J. E. Demuth, Phys. Rev. B 30, 5968 (1984).

[24] M.-L. Xu, B. M. Hall, S. Y. Tong, M. Rocca, H. Ibach, S. Lehwald, and J.E. Black, Phys. Rev. Lett. 54, 1171 (1985).

[25] See Supplemental Material at http://link.aps.org/ supplemental/10.1103/PhysRevLett.113.186804, which includes Ref. [26].

[26] S. Y. Tong, C. H. Li, and D. L. Mills, Phys. Rev. Lett. 44, 407 (1980).

[27] K. Schouteden, P. Lievens, and C. Van Haesendonck, Phys. Rev. B 79, 195409 (2009).

[28] R. Mazzarello, A. Dal Corso, and E. Tosatti, Surf. Sci. 602, 893 (2008).

[29] U. Krieg, C. Brand, C. Tegenkamp, and H. Pfnür, J. Phys. Condens. Matter 25, 014013 (2013). 